\title{
GW23-e0427 EFFECT OF AUTOLOGOUS BONE MARROW MONONUCLEAR CELLS TRANSPLANTATION IN DIABETIC PATIENTS WITH ST-SEGMENT ELEVATION MYOCARDIAL INFARCTION
}

doi:10.1136/heartjnl-2012-302920k.7

Dongdong Sun, Haichang Wang, Feng Cao. Department of Cardiology, Xijing Hospital, Fourth Military Medical University

Objectives To investigate the efficacy and proposed mechanism of bone marrow mononuclear cells (BMMNCs) transplantation for diabetic and non-diabetic patients with ST-segment elevation myocardial infarction (STEMI).

Methods One hundred and sixteen patients with STEMI who had successfully undergone percutaneous coronary intervention (PCI) were divided into a diabetic group $(\mathrm{n}=51)$ and non-diabetic group $(n=65)$. All of the patients received intracoronary injection of BMMNCs.

Results Diabetes down-regulated IGF-1, IGFBP-5, VEGF, SDF-1, IL6 , IL- $1 \alpha$ and TNF- $\alpha$ expression and affected the expression of Bmi1, Gfi1, Tel and Hox-B4 which could prevent premature senescence and maintain the self-renewal capacity of stem cells. Event-free survival rates were not statistically different between the diabetic and non-diabetic group ( $80 \%$ vs $72.5 \%, p=0.382)$. LV ejection fraction (LVEF) and wall motion score index (WMSI) were evaluated by echocardiography and found to be significantly improved in the non-diabetic group compared to the diabetic group over the 4-year 


\section{ABSTRACTS}

follow-up period. Improved myocardial perfusion and reduced infarct size in the non-diabetic group compared to the diabetic group was verified using single-photon emission computed tomographic (SPECT) imaging. The non-diabetic group also had reduced anginal symptoms as assessed by changes in their Seattle Angina Questionnaire scores and Canadian Cardiovascular Society (CCS) Functional Angina classification. An improvement of 6-min walk distance (6MWD) was also noted to be higher in the non-diabetic group during the follow-up period.

Conclusions This study indicates that the beneficial effect of BMMNCs transplantation for STEMI is less pronounced in diabetic patients. The mechanism is associated with decreased BMMNCs function in diabetic patients. 